Review

\title{
Function of Glia in Aging and the Brain Diseases
}

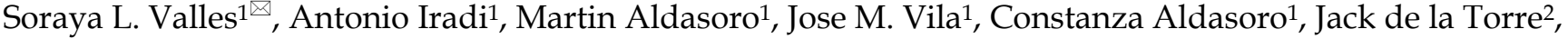 \\ Juan Campos-Campos ${ }^{3}$, and Adrian Jorda 1,3 \\ 1. Department of Physiology, School of Medicine, University of Valencia, Spain. \\ 2. University of Texas, Austin, Texas, USA. \\ 3. Department of Nursing, Faculty of Nursing and Podiatry, University of Valencia, Spain. \\ $\triangle$ Corresponding author: Soraya Valles, Department of Fisiologia, Facultad de Medicina, Av. Blasco Ibanez 15, Universidad de Valencia, Valencia 46010, Spain, \\ Tel: 34963983813, Fax: 34963864642, E-mail: lilian.valles@uv.es. \\ (1) The author(s). This is an open access article distributed under the terms of the Creative Commons Attribution License (https://creativecommons.org/licenses/by/4.0/). \\ See http://ivyspring.com/terms for full terms and conditions.
}

Received: 2019.06.20; Accepted: 2019.08.18; Published: 2019.10.05

\begin{abstract}
Microglia cells during aging, neurodegeneration and neuroinflammation show different morphological and transcriptional profiles (related to axonal direction and cell adhesion). Furthermore, expressions of the receptors on the surface and actin formation compared to young are also different. This review delves into the role of glia during aging and the development of the diseases. The susceptibility of different regions of the brain to disease are linked to the overstimulation of signals related to the immune system during aging, as well as the damaging impact of these cascades on the functionality of different populations of microglia present in each region of the brain. Furthermore, a decrease in microglial phagocytosis has been related to many diseases and also has been detected during aging. In this paper we also describe the role of glia in different illness, such as AD, ALS, pain related disorders, cancer, developmental disorders and the problems produced by opening of the blood brain barrier. Future studies will clarify many points planted by this review.
\end{abstract}

Key words: Aging, brain diseases, glia.

\section{Microglia and Aging}

The aged microglia of the human cerebral cortex to show different morphological abnormalities, including, spherical and knotty shapes, less branching, as well as fragmentation processes (1). Microglia isolated from post-mortem samples of parietal cortex in elderly people also show different transcriptional profiles from cells obtained in young subjects whose genes are associated with cell adhesion, axonal direction, receptor expression on their surfaces and actin formation where they were especially affected (2). Grabert and his group (3) found that changes at the genetic level related to the immune system, and to a lesser extent the genes related to cellular bioenergy, were widely associated with the diversity related to the brain region and its age (3).

Microglia are also greatly affected by aging and disease at the molecular level. Using "high- dimensional single-cell proteomic mapping" techniques, Mrdjen et al., (4), identified diverse populations of inflammatory cells, including microglia, in the adult mouse brain. In the same way, it has also been possible to find remarkable changes and the proteomic signature of these cells during aging, neurodegeneration and neuroinflammation. Compared with cells from young mice, a population of aged microglia expresses higher levels of phagocytosis associated with the CD11c and CD14 markers. In addition, different populations of microglia were identified in the brains of old mice, where the reactive population expressed higher levels of CD11c and CD14, CD86, CD44, programming ligand of death 1 and MHC-II, and lower levels of markers of microglial homeostasis CX3CR1, MerTK (C-MER protooncogen tyrosine kinase), and Siglec-H, compared with non-reactive microglia (4). TNF-a, 
IL-1 $\beta$, and IL-6 are among the proinflammatory cytokines that are overproduced during aging. Cribbs and his group (5) suspected that this was caused by overstimulation of the transcription factor NF-KB when the microglia underwent cell senescence (5). Biochemically, aged microglia produce more reactive oxidative species and inflammatory cytokines (6). In the same way, a positive relationship between these oxidative species and inflammatory cytokines has also been found in the brain and spinal cord of the APP/PS1KI mouse model of Alzheimer's disease (7).

The overproduction of proinflammatory mediators leads to microglia sensitization, or age-related microglial priming, so that aged microglia produce an exaggerated but inefficient response to inflammatory stimuli. Wendeln et al. (8) recently demonstrated that peripheral stimulation of mouse microglial cells by repeated injection of LPS can cause epigenetic changes in these cells for more than six months (8). This suggests that the basal level of inflammation in microglia can be increased with repetitive inflammatory stimuli, potentially being the cause of microglial priming. Cumulative and lasting changes in the balance of inflammatory mediators worsen the ability of microglia to perform basic physiological functions and are probably a contributing factor in neurodegenerative processes. For example, young microglia can more efficiently phagocytose disease-related proteins, such as $A \beta$ (9) and a-synuclein (10) than aged microglia. Similarly, it has been shown that aged microglia decrease motility, vigilance, and phagocytic responses to demyelinating lesions (11), in part due to reduced lysosomal function (12). Taken together, these studies suggest that the susceptibility of different regions of the brain to Alzheimer's disease (AD) may be linked to the overstimulation of signals related to the immune system during aging, as well as the damaging impact of these cascades on the functionality of different populations of microglia present in each region of the brain.

\section{Decrease in Microglial Phagocytosis during Aging}

As the main phagocytic cells in the brain, microglia play a central role in the clearance of $A \beta$. However, the effectiveness of this clearance decreases during aging, and particularly in AD (9). Despite its inability to clear $A \beta$ from the brain, microglia continue to release proinflammatory mediators to further stimulate the immune response, thus creating a cycle that leads to the accumulation of activated immune cells, inflammatory mediators and $A \beta$. This cycle which is partially caused by microglia senescence has been reported by Thakker et al. (13) and can be reversed by blocking $A \beta$ synthesis. (13). The microglia of old APP / PS1 mice exhibit lower expression of the scavenger receptor A (for its acronym in English SRA), CD36 and the receptor for RAGE (three scavenger receptors for beta-amyloid binding) when compared to that observed in the cells from young mice. By contrast, microglia from the group of APP / PS1 mice expresses higher levels of the proinflammatory cytokines, IL-1 $\beta$ and TNF- $\alpha$, suggesting that there is an inverse correlation between the production of pro-inflammatory cytokines and clearance of $A \beta$. This idea is supported by in vitro studies in which treatment of microglia with TNF- $\alpha$ resulted in a reduction of the expression of SRA and CD36, and uptake of $\mathrm{A} \beta$ (14). In AD mouse models, microglia also show substantial deterioration in calcium signalling (15) and beclin-1 mediated recycling of phagocytic receptors CD36 and Trem2 (16), which are linked to a poor internalization of $\mathrm{A} \beta$.

Based on longitudinal studies of images of the human brain, Fan et al. indicated the state of microglia activation changes from an early protective phenotype to a late and noxious phenotype during the progression of $\mathrm{AD}$ (17). The chronic activation of different populations of microglia could be associated with the change in the global microglial phenotype depending on whether they are CX3CR1 + for the production of inflammatory mediators or Trem $2+$ for phagocytosis of $A \beta(18)$.

Although there is strong evidence that aging impairs microglial activity, the relationship between microglial senescence, $\mathrm{A} \beta$ and $\mathrm{AD}$ remains incomplete, given that some studies have shown that microglial phagocytic activity towards $A \beta$ is not necessarily related to changes in neurotoxicity and cognition. Investigations using the APP J20 mouse model have revealed that the inhibition of microglial phagocytic activity by minocycline before the accumulation of $A \beta$ results in an increase in amyloid plaque burden, reduced inflammation, and improves cognitive performance, which indicates that chronic inflammation can interrupt normal neuronal function independent of $A \beta$. However, when microglial inhibition is performed after $A \beta$ deposition begins, inflammation is suppressed by minocycline with no effect on amyloid plaque loading or in the improvement in cognitive performance (19). To add even more controversy to the relationship between inflammation and $\mathrm{AD}$, the pharmacological or genetic suppression of microglia after $A \beta$ accumulation does not change the plaque levels but prevents dendritic loss of the vertebral spine, neuronal loss and improves cognitive performance $(20,21)$. 


\section{Alzheimer's Disease and Glia}

In Alzheimer's disease, complex changes and specific conflicts occur in different brain regions. The number of reactive astrocytes increases, engulfing and reducing the amyloid plaques. In addition, astrocytes surround the amyloid plaques and secrete proinflammatory factors in the extracellular space (22). Currently, no single hypothesis about what causes AD has been proven, a fact which argues for the heterogeneity of this dementia. For decades, it has been thought by many that the amyloid cascade hypothesis was the correct cause and this thinking was supported and promoted financially by a host of pharmaceutical companies around the world. The $A \beta$ hypothesis argues that in AD there is an increase in oxidative stress caused by the accumulation of $A \beta$ and that its elimination has been a priority but without positive results to the patients. There are much researches showing that increased levels of ROS have been linked to AD (23) but the effects of antioxidants in clinical studies have been disappointing, either because high concentrations of antioxidants are pro-oxidants, or because oxidative stress occurs relatively early in the course of the disease, or, because the combination of antioxidants fails in the clinical stage. Analogous to microglia, astrocytes play multiple roles in the organization and maintenance of brain structure and function. Multiple studies show that astrocytes dynamically modulate information processing, signal transmission, neural and synaptic plasticity, as well as homeostasis control of the blood-brain barrier. The astrocytic role in immune responses is not entirely clear. The evidence suggests that, astrocytes act as a protector during cerebral ischemia, whereas against inflammation mediated by the lipopolysaccharide of Escherichia coli, its intervention seems to be harmful (24). In the cells of the retina, however, it has been reported that through the production of lipoxins, astrocytes have an anti-inflammatory and neuroprotective effect against acute and chronic lesions (25). Similarly, the role of the cytokine IL-33 produced by astrocytes has recently been demonstrated to the microglial approach to the synaptic terminals, as well as the development of neural circuits (26). From prior studies discussed above on the action of IL-1 $\alpha^{+}$, it may be concluded that there is also a correlation between IL-1a and the greater number of GFAP + astrocytes (GFAP-immunoreactive astrocytes) (27). Conversely, it has been demonstrated in a study carried out in mice with multiple sclerosis that signals of the proinflammatory cytokine TNF- $\alpha$ promote in astrocytes a change in synaptic transmission and produce interference at the cognitive level (28). Other studies have shown that the activation of certain transcription factors are also involved in protective (STAT3) (29) or injurious (NF-kB) effects (30). More details about the relationships between glia cells and Alzheimer's disease can find them in (31).

\section{Amyotrophic Lateral Sclerosis (Als) and Glia}

In ALS cortical and spinal motor neurons suffer damage, sequentially or simultaneously. Until 25 years ago, astrocytes were only considered the architecture of the CNS. Since then, many other functions have been described for these cells. The astrocytes adapt their function to the needs of neurons associated with them, and vary according to the mission they need to perform $(32,33)$. The population of astrocytes connected to a neuron group have defined functions and morphological characteristics that are different from other astrocytic populations. Fibrous astrocytes have a fusiform body located in parallel with nerve fibers and produce extensions even directly on the nodes of Ranvier. $(32,34)$. Each astrocyte controls a space with several motor neurons and makes contact with 3 to 10 neurons, hundreds of dendrites and with multiple synapses (35). Astrocytes connect to each other through gap-junctions (GJs). The GJs do the intercellular communication through ion exchange (electrical coupling) and the passage of small molecules (metabolic coupling) develops a type of syncytium that extends across the CNS. Therefore, all astrocytes in the brain constitute a network that helps neurons and other types of brain cells in the brain, controlling inflammation, oxidative stress, and brain nutrition (36). Furthermore, astrocytes control microglia and the inflammatory reaction produced by macrophages. The GJs communicate with protoplasmic and fibrous astrocytes, with neurons and their extensions as well as with arterioles (37). Motor neuron death, which occurs through mitochondrial, cytoplasmic apoptosis or vacuolization $(38,39)$, can affect neurons by noxious circumstances. Astrocytes are also important because any alteration that affects cortical or spinal neurons will cause consequences in neurons far removed. However, astrocytes have different populations surrounding neuron types and that is true for protoplasmic astrocytes, which make contact with neurons, and for fibrous astrocytes that contact neuronal extensions $(40,41)$. A recent paper, published by Larrodé et al. (42), demonstrated the involvement of DREAM (Downstream Regulatory Element Antagonist Modulator) in ALS. This protein could be used as a novel therapeutic target to treat ALS, but further studies are needed to understand the molecular mechanism of DREAM in ALS.

ALS begins within a group of cortical and/or 
spinal neurons, and protoplasmic astrocytes may send toxic mediators to other protoplasmic cells surrounding different neuronal groups. Furthermore, protoplasmic astrocytes are normally in communication with fibrous astrocytes connecting that with axons of the pyramidal cells (43). It is possible that these protoplasmic astrocytes could induce lethal damage in the cells implicated.

\section{Glia and Its Role in Pain}

Pain plays a protective body role that functions as a signal of damage. Pain can convert into a disease when it persists more than 3 or 4 months (chronic pain) (44). In the sensory ganglia, particularly in the dorsal spinal ganglia, there are different types of cells. Cultures of the dorsal spinal ganglia in the rat demonstrate a presence of organized cells, seen using light and electron microscopy. These cells surround each body and the proximal portion of a neuronal axon forming a sheath around the cell. Each unit is a morphological and functional distinct body (45) and every unit is locked on with a neighbor by adhesion and gap junctions. Satellite glial (SG) cells are important in non-physiological pain and are a potential target for the development of new pain treatments (46). The cells in the sensory ganglia are surrounded by neuronal bodies presenting different functional units with bidirectional communication signaling between both types of neural cells. SG cells develop biochemical and structural changes after nerve damage, and consequently, the development of pain in animal models $(47,48)$. A somatic release of chemical mediators, dependent on $\mathrm{Ca}^{2+}$, occurs after electric or chemical stimulation which alter somatic excitability in the sensory ganglia (49), such as substance $\mathrm{P}$, glutamate, adenosine triphosphate, $\gamma$-amino-butyric acid and CGRP (calcitonin gene related peptide) $(50,51)$.

The communication between cells in the sensory ganglia (SG), neuron-neuron; neuron-SG or SG-SG, can affect the communication between cells. The SG cells can modulate chronic pain $(52,53)$ and sensory ganglia can be the pathophysiological first level of chronic pain. Furthermore, interaction between SG cells and neurons is becoming more and more important in the therapeutic field of chronic pain.

\section{Glia and Cancer}

Tumor types are determined by age, size and location. In infancy and adolescence, infratentorial astrocytoma and midline tumors such as medulloblastoma and pinealoma predominate. In adulthood, anaplastic astrocytoma and glioblastoma are more common (54). Glioblastoma is the most common tumor detected in the brain, although the most frequent tumor seen at autopsy is meningioma. Secondary glioblastomas progress from low-grade diffuse astrocytoma or anaplastic astrocytoma (55). Some brain tumors, schwannoma, sarcoma, glioma and meningioma, persist after the patient has been exposed to chemotherapy and/or radiation therapy. In Epstein Barr virus and in primary brain lymphoma, virus infections are involved and in many cases, they are associated with AIDS.

Astrocytes are strongly associated with cancer in the brain (56) as cancerous astrocytoma. There are three different astrocytoma types, pilocytic, diffuse and anaplastic astrocytoma. Pilocytic astrocytoma is always treated by surgery and is more prevalent in children (57). In diffuse astrocytoma, surgery and radiotherapy is generally recommended. In anaplastic astrocytoma the same treatment as that for glioblastoma is used (indicated below) (58). Investigation of astrocytomas has been studied with the use of anti-cancer stem cell drugs (59). Another type of cancer is glioblastoma multiform which represents $20 \%$ of all intracranial tumors, constituting half of all glioblastomas. They grow from tissue surrounding nerve cells and normally start in the cerebral hemispheric part of the brain. It affects $60 \%$ of men and $40 \%$ of women. They have high malignancy, producing quick invasion of the brain tissue (60). Because of their malignancy, natural and synthetic drugs have been used (61). Targeting cholesterol metabolism has also been used in cancer therapy to control changes in aberrant cholesterol production in neuroblastoma (62).

Non-neuron cells can develop cancer, such as astrocytes, and oligodendroglia. The neurons are differentiated cells, so they cannot produce cancer. The neuroblastoma cells are radial glia or precursors of astrocytes that can develop before their differentiation to neurons. Glial cells develop different types of cells such as neurons, oligodendroglia and astrocytes (63). All these types of cells can convert to cancer and affect the normal function of the brain). Radial glia are stem cells that develop from a progenitor stem cell in the embryo and in the adult brain (64). It is of interest to point out that in some astrocytomas, the patient may increase cognitive capacity and memory, before the illness starts and even when cancer is already present (65).

\section{Glia and Developmental Disorders}

Astrocytes play a role in the aberrant formation of neuronal circuitry in the brain. This field in neuroscience is relatively new and shows us the involvement of astrocytes in psychiatric disorders, including autism spectrum disorders (ASDs), bipolar disorders (BD) and schizophrenia $(66,67)$. The 
expression of the MicroRNAs are critical in the development of prefrontal cortical circuitry during the postnatal brain development. Deficits in neuronal maturation produced by the microRNAs decrease and/or become deficient and can be demonstrated in some neuropsychiatric diseases (67). Coordination of the different neural cell types is fundamental in the development of the neural network and in normal neural function. Astrocytes coordinate neural development by controlling the formation of synapses, neuronal function, driving axon growth, and promoting neuronal survival (68). Astrogenesis occurs after neurons develop in the brain of rats (64), and in other animals, including humans (69). During development of the brain, chromatin changes occur and defects in astrogenesis or in early function of astrocytes that can produce the progression of neurodevelopmental disorders (68).

\section{The Opening of the Blood-Brain Barrier during Aging}

Another characteristic of aging with relevance for $\mathrm{AD}$ is the increase in the permeability of the blood-brain barrier (BBB) to immune cells and the peripheral tissue molecules. The loss of integrity of the BBB appears to occur prior to hippocampal atrophy $(70,71)$ and cognitive impairment (72), suggesting that this decomposition precedes the neurodegenerative process in AD. This evidence also indicates that the peripheral activation of the immune system may contribute to the deterioration of brain function and the neurodegenerative processes that occur in $\mathrm{AD}$ (73). Interestingly, an association has recently been made between inflammation in middle-aged individuals and brain volume in older individuals. Compared with middle-aged people without elevated inflammatory markers, individuals with elevations in 3 or more markers had, on average, $5 \%$ more decreased of the volume hippocampus, typical of the AD. (71). In healthy individuals, those responsible for the proper functioning of neurovascular function are the pericytes, endothelial cells and astrocytes, which form fine junctions between endothelial cells in response to inflammation that limit the consequent mobilization of immune cells from the periphery. It has been found that inhibiting the formation of the inducible astrocytic barrier increases the severity of the disease in mouse models. In this way, it is suspected that the senescence of the cellular components that form this barrier is one of the causes behind the malfunction of the BBB (74).

Endothelial cells, pericytes and astrocytes in the BBB are particularly vulnerable to the effects of aging and chronic stimulation by inflammatory mediators. During aging, endothelial cells in the mouse brain express higher levels of TNF-a and decreased expression of the narrow-binding proteins ocludin-1 and zonula occludens1, which correlate with an increase in peripheral inflammation (75). Aging and, more aggressively, $\mathrm{AD}$, also cause damage to the pericytes as evidenced by the increase in $\beta$-platelet-derived growth factor receptor levels observed in experiments performed on mice (70). Although the mechanism of induction of pericyte lesions is still unknown, Bell and his collaborators have shown that age-dependent vascular damage in pericyte-deficient mice precedes neuronal degenerative changes, learning and memory impairment, and neuroinflammatory response (76).

In astrocytes, transcriptome analysis has revealed that aging induces upregulation of several genes related to the immune system (77). An age-dependent change in the phenotype of the astrocytes was identified by comparing gene expression in the astrocytes of 10-week and 2-year-old mice using RNAseq. It has been demonstrated that IL-1 $\beta$ suppresses the astrocytic expression of "sonic hedgehog" (78), a protein that protects the BBB by upregulation of the tight binding proteins in capillary endothelial cells (79). Similarly, this study demonstrated that astrocytes from healthy 2-year-old mice expressed genetic markers that correspond to the activated A1 phenotype, including C4a, C3, Serpina3n, and Cxcl10 (80). IL-1 $\beta$ also increases the production of proinflammatory chemokines such as CCL2, CCL20 and CXCL2 by astrocytes, which induces the migration of immune cells from the periphery and exacerbates BHE malfunction and neuroinflammation (78). Therefore, an excessive proinflammatory phenotype significantly alters the protective role of astrocytes in the maintenance of BBB integrity.

Inflammation and aging are therefore closely linked thanks to studies suggesting that low levels of inflammation correlate better with healthy brain function (71) and longevity (81). Considering that most cells in the brain, including astrocytes (30) and microglia (82), have a long lifespan, it is plausible that the accumulation and overstimulation of Inflammation can trigger multiple cumulative molecular modifications (telomere shortening, DNA damage, epigenetic modifications, lysosomal dysregulation) that eventually contribute to cellular senescence and loss of function. This idea is, at least in part, supported by a repopulation study in a neurodegeneration model of mice, which showed that, following a pharmacologically induced cell depletion, the microglia that repopulated the brain showed the morphological phenotype of young cells. Surprisingly, the animals also showed a significant 
improvement in brain function (83). It is still unknown that cell repopulation methods can restore the molecular signatures of immunesenescence. Additional studies are needed to clarify the underlying cellular and molecular mechanisms related to the immune dysregulation that occurs during aging that divert individuals from the relatively benign process of normal brain aging to the pathological processes associated with AD.

\section{Conclusion}

The functions that the glia develops, both during the age and the illness, is an important field of study at present. The most important brain cells can be divided into neurons and glia. For many years research has focused on neurons, but increasingly the glia has becoming more important. Within the glia are the astrocytes, oligodendroglia and microglia. Many diseases affect one or several of these cells. From Alzheimer's disease, amyotrophic lateral sclerosis, pain-related diseases, cancer and developmental disorders, we know now that glia play an important role in the development of their diseases. Studies are needed to clarify the underlying cellular and molecular mechanisms related to the immune dysregulation that occurs during aging that divert individuals from the relatively benign process of normal brain aging to the pathological processes associated with different diseases.

\section{Competing Interests}

The authors have declared that no competing interest exists.

\section{References}

1. Streit WJ, Mrak RE, Griffin WS. Microglia and neuroinflammation: a pathological perspective. J Neuroinflammation. 2004;1:14.

2. Galatro TF, Holtman IR, Lerario AM, Vainchtein ID, Brouwer N, Sola PR, et al. Transcriptomic analysis of purified human cortical microglia reveals age-associated changes. Nat Neurosci. 2017;20:1162-1171.

3. Grabert K, Michoel T, Karavolos MH, Clohisey S, Baillie JK, Stevens MP, et al. Microglial brain region-dependent diversity and selective regional sensitivities to aging. Nat Neurosci. 2016;19:504-16.

4. Mrdjen D, Pavlovic A, Hartmann FJ, Schreiner B, Utz SG, Leung BP, et al. High-Dimensional Single-Cell Mapping of Central Nervous System Immune Cells Reveals Distinct Myeloid Subsets in Health, Aging, and Disease. Immunity. 2018;48:380-395.

5. Cribbs DH, Berchtold NC, Perreau V, Coleman PD, Rogers J, Tenner AJ, et al. Extensive innate immune gene activation accompanies brain aging, increasing vulnerability to cognitive decline and neurodegeneration: a microarray study. J Neuroinflammation. 2012;9:179.

6. Ritzel RM, Crapser J, Patel AR, Verma R, Grenier JM, Chauhan A, et al. Age-Associated Resident Memory CD8 T Cells in the Central Nervous System Are Primed To Potentiate Inflammation after Ischemic Brain Injury. J Immunol. 2016;196:3318-30.

7. Wirths $\mathrm{O}$, Breyhan $\mathrm{H}$, Marcello A, Cotel MC, Brück W, Bayer TA. Inflammatory changes are tightly associated with neurodegeneration in the brain and spinal cord of the APP/PS1KI mouse model of Alzheimer's disease. Neurobiol Aging. 2010;31:747-57.

8. Wendeln AC, Degenhardt K, Kaurani L, Gertig M, Ulas T, Jain G, et al. Innate immune memory in the brain shapes neurological disease hallmarks. Nature. 2018:556:332-338.

9. Njie EG, Boelen E, Stassen FR, Steinbusch HW, Borchelt DR, Streit WJ. Ex vivo cultures of microglia from young and aged rodent brain reveal age-related changes in microglial function. Neurobiol Aging. 2012;33:195.
10. Bliederhaeuser C, Grozdanov V, Speidel A, Zondler L, Ruf WP, Bayer H, et al. Age-dependent defects of alpha-synuclein oligomer uptake in microglia and monocytes. Acta Neuropathol. 2016;131:379-91.

11. Sun M, Brady RD, Casillas-Espinosa PM, Wright DK, Semple BD, Kim HA, et al. Aged rats have an altered immune response and worse outcomes after traumatic brain injury. Brain Behav Immun. 2019 Apr 27. pii: S0889-1591(18)30850-X

12. Safaiyan S, Kannaiyan N, Snaidero N, Brioschi S, Biber K, Yona S, et al. Age-related myelin degradation burdens the clearance function of microglia during aging. Nat Neurosci. 2016;19:995-8.

13. Thakker DR, Weatherspoon MR, Harrison J, Keene TE, Lane DS, Kaemmerer $\mathrm{WF}$, et al. Intracerebroventricular amyloid-beta antibodies reduce cerebral amyloid angiopathy and associated micro-hemorrhages in aged Tg2576 mice. Proc Natl Acad Sci U S A. 2009;106:4501-6.

14. Hickman SE, Allison EK, El Khoury J. Microglial dysfunction and defective beta-amyloid clearance pathways in aging Alzheimer's disease mice. J Neurosci. 2008;28:8354-60.

15. Brawek B, Garaschuk O. Network-wide dysregulation of calcium homeostasis in Alzheimer's disease. Cell Tissue Res. 2014;357:427-38.

16. Lucin KM, O'Brien CE, Bieri G, Czirr E, Mosher KI, Abbey RJ, et al. Microglial beclin 1 regulates retromer trafficking and phagocytosis and is impaired in Alzheimer's disease. Neuron. 2013;79:873-86.

17. Fan Z, Brooks DJ, Okello A, Edison P. An early and late peak in microglial activation in Alzheimer's disease trajectory. Brain. 2017;140:792-803.

18. Keren-Shaul H, Spinrad A, Weiner A, Matcovitch-Natan O, Dvir-Szternfeld R, Ulland TK, et al. Unique Microglia Type Associated with Restricting Development of Alzheimer's Disease. Cell. 2017;169:1276-1290.

19. Seabrook TJ, Jiang L, Maier M, Lemere CA. Minocycline affects microglia activation, Abeta deposition, and behavior in APP-tg mice. Glia. 2006;53:776-82.

20. Olmos-Alonso A, Schetters ST, Sri S, Askew K, Mancuso R, Vargas-Caballero $\mathrm{M}$, et al. Pharmacological targeting of CSF1R inhibits microglial proliferation and prevents the progression of Alzheimer's-like pathology. Brain. 2016;139:891-907.

21. Spangenberg EE, Green KN. Inflammation in Alzheimer's disease: Lessons learned from microglia-depletion models. Brain Behav Immun. 2017;61:1-11.

22. Farina E, Baglio F, Pomati S, D'Amico A, Campini IC, Di Tella S, et al. The Mirror Neurons Network in Aging, Mild Cognitive Impairment, and Alzheimer Disease: A functional MRI Study. Front Aging Neurosci. 2017;9:371.

23. Markesbery WR. The role of oxidative stress in Alzheimer disease. Arch Neurol. 1999;56:1449-1152.

24. Zamanian JL, Xu L, Foo LC, Nouri N, Zhou L, Giffard RG, et al. Genomic analysis of reactive astrogliosis. J Neurosci. 2012;32:6391-410.

25. Livne-Bar I, Wei J, Liu $\mathrm{HH}$, Alqawlaq $\mathrm{S}$, Won GJ, Tuccitto A, et al. Astrocyte-derived lipoxins A4 and B4 promote neuroprotection from acute and chronic injury. J Clin Invest. 2017;127:4403-4414.

26. Vainchtein ID, Chin G, Cho FS, Kelley KW, Miller JG, Chien EC, et al Astrocyte-derived interleukin-33 promotes microglial synapse engulfment and neural circuit development. Science. 2018;359:1269-1273.

27. Sheng JG, Mrak RE, Griffin WS. Microglial interleukin-1 alpha expression in brain regions in Alzheimer's disease: correlation with neuritic plaque distribution. Neuropathol Appl Neurobiol. 1995;21:290-301.

28. Habbas S, Santello M, Becker D, Stubbe H, Zappia G, Liaudet N, et al. Neuroinflammatory TNFa Impairs Memory via Astrocyte Signaling. Cell. 2015;163:1730-41.

29. Anderson MA, Burda JE, Ren Y, Ao Y, O'Shea TM, Kawaguchi R, et al. Astrocyte scar formation aids central nervous system axon regeneration. Nature. 2016:532:195-200.

30. Liddelow SA, Guttenplan KA, Clarke LE, Bennett FC, Bohlen CJ, Schirmer L, et al. Neurotoxic reactive astrocytes are induced by activated microglia. Nature. 2017:541:481-487.

31. Perez-Nievas BG, Serrano-Pozo A. Deciphering the Astrocyte Reaction in Alzheimer's Disease. Front Aging Neurosci. 2018;10:114.

32. Kimelberg HK. Functions of mature mammalian astrocytes: a current review. Neuroscientist. 2010;16:79-106.

33. Reichenbach A, Derouiche A, Kirchhoff F. Morphology and dynamics of perisynaptic glia. Brain Res Rev. 2010;63:11-25.

34. Hewett JA. Determinants of regional and local diversity within the astroglial lineage of the normal central nervous system. I Neurochem. 2009;110: 1717-1736.

35. Bogaert E, d'Ydewalle C, Van Den Bosch L. Amyotrophic lateral sclerosis and excitotoxicity: from pathological mechanism to therapeutic target. CNS Neurol Disord Drug Targets. 2010;9:297-304.

36. Araque A, Carmignoto G, Haydon PG. Dynamic signaling between astrocytes and neurons. Annu Rev Physiol. 2001;63:795-813.

37. Alonso A, Reinz E, Jenne JW, et al. Reorganization of gap junctions after focused ultrasound blood-brain barrier opening in the rat brain. J Cereb Blood Flow Metab. 2010;30:394-402.

38. Gonzalez Deniselle MC, Lopez-Costa JJ, Saavedra JP, et al. Progesterone neuroprotection in the Wobbler mouse, a genetic model of spinal cord motor neuron disease. Neurobiol Dis. 2002:11:457-68.

39. Valles SL, Borrás C, Gambini J, Furriol J, Ortega A, Sastre J, et al. Oestradiol or genistein rescues neurons from amyloid beta-induced cell death by inhibiting activation of p38. Aging Cell. 2008;7:112-8. 
40. Yamamoto T, Ochalski A, Hertzberg EL, Nagy JI. On the organization of astrocytic gap junctions in rat brain as suggested by LM and EM immunohistochemestry of connexin43 expression. J Comp Neurol. 1990;302:853-83.

41. Pereira A Jr, Furlan FA. Astrocytes and human cognition: modelling information and modulation of neuronal activity. Prog Neurorobiol. 2010;92:405-20.

42. Larrodé P, Calvo AC, Moreno-Martínez L, de la Torre M, Moreno-García L, Molina N, et al. DREAM-Dependent Activation of Astrocytes in Amyotrophic Lateral Sclerosis. Mol Neurobiol. 2018;55:1-12

43. Sica RE. Esclerosis lateral amiotrófica: ¿es el astrocito la célula primariamente dañada? MEDICINA (Buenos Aires). 2013;73:573-578.

44. Scholz J, Woolf CJ. Can we conquer pain? Nat Neurosci. 2002;5:1062-1067.

45. Hanani M. Satellite glial cells in sensory ganglia: from form to function. Brain Res Brain Res Rev. 2005;48:457-76.

46. Leite Costa FA and Moreira Neto FL. Células gliales satélite de ganglios sensoriales: su papel en el dolor. Rev Bras Anestesiol. 2015;65:73-81

47. McMahon SB, Malcangio M. Current challenges in glia-pain biology. Neuron. 2009;64:46-54

48. Hanani M. Satellite glial cells: more than just 'rings around the neuron. Neuron Glia Biol. 2010;6:1-2.

49. Huang LY, Neher E. Ca(2+)-dependent exocytosis in the somata of dorsal root ganglion neurons. Neuron. 1996;17:135-145.

50. Matsuka Y, Neubert JK, Maidment NT, et al. Concurrent release of ATP and substance $\mathrm{P}$ within guinea pig trigeminal ganglia in vivo. Brain Res. 2001;915:248-155.

51. McCarthy PW, Lawson SN. Differing action potential shapes in rat dorsal root ganglion neurons related to their substance $P$ and calcitonin gene-related peptide immunoreactivity. J Comp Neurol. 1997;388:541-549.

52. Ohara PT, Vit JP, Bhargava A, et al. Gliopathic pain: when satellite glial cells go bad. Neuroscientist. 2009;15:450-463.

53. Ledda M, Blum E, de Palo S, et al. Augmentation in gap junction mediated cell coupling in dorsal root ganglia following sciatic nerve neuritis in the mouse. Neuroscience. 2009;164:1538-1545.

54. Raucher D. Tumor targeting peptides: novel therapeutic strategies in glioblastoma. Curr Opin Pharmacol. 2019;47:14-19.

55. Ohgaki $\mathrm{H}$, Kleihues $\mathrm{P}$. The definition of primary and secondary glioblastoma. Clin Cancer Res. 2013;19:764-72.

56. Tomiyama A, Kobayashi T, Mori K, Ichimura K. Protein Phosphatases-A Touchy Enemy in the Battle Against Glioblastomas: A Review. Cancers (Basel). 2019;11. pii: E241. doi:10.3390/cancers11020241.

57. Benavides-Varela S, Lorusso R, Baro V, Denaro L, Estévez-Pérez N, Lucangeli $\mathrm{D}$, et al. Mathematical skills in children with pilocytic astrocytoma. Acta Neurochir (Wien). 2019;161:161-169.

58. Sokol E, Desai AV. The Evolution of Risk Classification for Neuroblastoma. Children (Basel). 2019;6. pii: E27. doi:10.3390/children6020027.

59. Kuramoto K, Yamamoto M, Suzuki S, Sanomachi T, Togashi K, Seino S, et al. AS602801, an Anti-Cancer Stem Cell Drug Candidate, Suppresses Gap-junction Communication Between Lung Cancer Stem Cells and Astrocytes. Anticancer Res. 2018;38:5093-5099.

60. Batash R, Asna N, Schaffer P, Francis N, Schaffer M. Glioblastoma Multiforme, Diagnosis and Treatment; Recent Literature Review. Curr Med Chem. 2017:24:3002-3009.

61. Ivanov VN, Wu J, Wang TJC, Hei TK. Inhibition of ATM kinase upregulates levels of cell death induced by cannabidiol and $\gamma$-irradiation in human glioblastoma cells. Oncotarget. 2019;25:825-846.

62. Pirmoradi L, Seyfizadeh N, Ghavami S, Zeki AA, Shojaei S. Targeting cholesterol metabolism in glioblastoma: a new therapeutic approach in cancer therapy. I Investig Med. 2019;14. pii: jim-2018-000962

63. Sancho-Tello M, Vallés S, Montoliu C, Renau-Piqueras J, Guerri C. Developmental pattern of GFAP and vimentin gene expression in rat brain and in radial glial cultures. Glia. 1995;15:157-66.

64. Valles S, Sancho-Tello M, Miñana R, Climent E, Renau-Piqueras J, Guerri C. Glial fibrillary acidic protein expression in rat brain and in radial glia culture is delayed by prenatal ethanol exposure. J Neurochem. 1996;67:2425-33.

65. Kuramoto K, Yamamoto M, Suzuki S, Sanomachi T, Togashi K, Seino S, et al. AS602801, an Anti-Cancer Stem Cell Drug Candidate, Suppresses Gap-junction Communication Between Lung Cancer Stem Cells and Astrocytes. Anticancer Res. 2018;38:5093-5099.

66. Pajarillo E., Rizor A., Lee J., Aschner M., Lee E. The role of astrocytic glutamate transporters GLT-1 and GLAST in neurological disorders: potential targets for neurotherapeutics. Neuropharmacology. 2019. pii: S0028-3908(19)30080-2. doi:10.1016/j.neuropharm.2019.03.002

67. Schofield CM, Hsu R, Barker AJ, Gertz CC, Blelloch R, Ullian EM. Monoallelic deletion of the microRNA biogenesis gene Dgcr8 produces deficits in the development of excitatory synaptic transmission in the prefrontal cortex. Neural Dev. 2011;6:11.

68. Sloan SA, Barres BA. Mechanisms of astrocyte development and their contributions to neurodevelopmental disorders. Curr Opin Neurobiol. 2014;27:75-81

69. Paavilainen T, Pelkonen A, Mäkinen ME, Peltola M, Huhtala H, Fayuk D, et al. Effect of prolonged differentiation on functional maturation of human pluripotent stem cell-derived neuronal cultures. Stem Cell Res. 2018;27:151-161.
70. Montagne A, Barnes SR, Sweeney MD, Halliday MR, Sagare AP, Zhao Z, et al. Blood-brain barrier breakdown in the aging human hippocampus. Neuron. 2015;85:296-302.

71. Walker JA, Low KA, Fletcher MA, Cohen NJ, Gratton G, Fabiani M. Hippocampal structure predicts cortical indices of reactivation of related items. Neuropsychologia. 2017;95:182-192.

72. Takechi R, Lam V, Brook E, Giles C, Fimognari N, Mooranian A, et al. Blood-Brain Barrier Dysfunction Precedes Cognitive Decline and Neurodegeneration in Diabetic Insulin Resistant Mouse Model: An Implication for Causal Link. Front Aging Neurosci. 2017;9:399.

73. Villeda SA, Luo J, Mosher KI, Zou B, Britschgi M, Bieri G, et al. The ageing systemic milieu negatively regulates neurogenesis and cognitive function. Nature. 2011;477:90-4.

74. Horng S, Therattil A, Moyon S, Gordon A, Kim K, Argaw AT, et al. Astrocytic tight junctions control inflammatory CNS lesion pathogenesis. J Clin Invest. 2017;127:3136-3151.

75. Elahy M, Jackaman C, Mamo JC, Lam V, Dhaliwal SS, Giles C, et al. Blood-brain barrier dysfunction developed during normal aging is associated with inflammation and loss of tight junctions but not with leukocyte recruitment. Immun Ageing. 2015;12:2.

76. Bell RD, Winkler EA, Sagare AP, Singh I, LaRue B, Deane R, et al. Pericytes control key neurovascular functions and neuronal phenotype in the adult brain and during brain aging. Neuron. 2010;68:409-27.

77. Orre M, Kamphuis W, Osborn LM, Jansen AHP, Kooijman L, Bossers K, et al. Isolation of glia from Alzheimer's mice reveals inflammation and dysfunction. Neurobiol. Aging. 2014;35: 2746-2760.

78. Wang Y, Jin S, Sonobe Y, Cheng Y, Horiuchi H, Parajuli B, et al. Interleukin-1beta induces blood-brain barrier disruption by downregulating Sonic hedgehog in astrocytes. PLoS One. 2014;9(10):e110024. doi: 10.1371/journal.pone.0110024

79. Alvarez JI, Dodelet-Devillers A, Kebir H, Ifergan I, Fabre PJ, Terouz S, et al. The hedgehog pathway promotes blood-brain barrier integrity and CNS immune quiescence. Science. 2011;334:1727-31.

80. Clarke LE, Liddelow SA, Chakraborty C, Munch AE, Heiman M, Barres BA. Normal aging induces A1-like astrocyte reactivity. Proc Natl Acad Sci U S A. 2018;115:E1896-E905.

81. Arai Y, Martin-Ruiz CM, Takayama M, Abe Y, Takebayashi T, Koyasu S, et al. Inflammation, but not telomere length, predicts successful ageing at extreme old age: a longitudinal study of semi-supercentenarians. EBioMedicine. 2015;2:1549-58.

82. Fuger $\mathrm{P}$, Hefendehl JK, Veeraraghavalu $\mathrm{K}$, Wendeln AC, Schlosser $\mathrm{C}$, Obermuller U, et al. Microglia turnover with aging and in an Alzheimer's model via long-term in vivo single-cell imaging. Nat Neurosci. 2017;20:1371-6.

83. Rice RA, Pham J, Lee RJ, Najafi AR, West BL, Green KN. Microglial repopulation resolves inflammation and promotes brain recovery after injury. Glia. 2017;65:931-44. 